\title{
CHAPTER 5 NON-THERMAL EMISSION FROM EARLY-TYPE BINARIES
}

\author{
Gregor Rauw* \\ Institut d'Astrophysique et de Géophysique, Université de Liège, \\ Allée du 6 Août 17, Bât B5c, 4000 Liège (Sart-Tilman), Belgium \\ rauw@astro.ulg.ac.be
}

\begin{abstract}
In this chapter, I review the properties of high-energy particles in the stellar winds of hot early-type stars. Relativistic electrons are responsible for the synchrotron radio emission observed from a subsample of these stars. Most of the objects in the latter category are found to be binaries and the collision between the stellar winds of the binary components is thought to play a crucial role in the acceleration of the relativistic electrons. The interplay between these high-energy electrons and the intense stellar radiation field could produce a substantial nonthermal emission at X-ray and $\gamma$-ray energies through inverse Compton scattering. Other mechanisms, such as $\pi^{0}$ decay might also contribute to the production of non-thermal emission from hot stars. These various effects could possibly account for some of the yet unidentified EGRET sources found to be correlated with $\mathrm{OB}$ associations in our Galaxy. Finally, I review recent results from XMM-Newton observations and discuss the prospects of forthcoming $\gamma$-ray observations with INTEGRAL and GLAST.
\end{abstract}

\section{Introduction}

Early-type stars of spectral types $\mathrm{O}$ and Wolf-Rayet (hereafter WR) have energetic, highly supersonic, winds associating huge mass loss rates (of order $\dot{\mathrm{M}} \sim 10^{-6}$ and $10^{-5} \mathrm{M}_{\odot} \mathrm{yr}^{-1}$ for O-type and WR stars respectively) and large terminal velocities $\left(v_{\infty} \sim 2000 \mathrm{~km} \mathrm{~s}^{-1}\right)$. When two such stars form a binary system, their winds collide: the resulting wind interaction region is limited by two hydrodynamical shocks, each shock facing one of the binary components. The compressed stellar winds in 
the interaction region are separated by a contact discontinuity set by ram pressure equilibrium.

The theoretical concept of colliding stellar winds was put forward by Prilutskii \& Usov (1976) and Cherepashchuk (1976). The first models based on a numerical solution of the differential equations of the hydrodynamics describing the phenomenon were presented by Lebedev \& Myasnikov (1988), Luo et al. (1990) and Stevens et al. (1992). For a general introduction to theoretical aspects of this process, we refer to the paper of Stevens et al. (1992).

Over the last two decades, ample observational evidence for colliding wind interactions has been accumulated. For instance, optical and ultraviolet spectra of many early-type binaries display phase-locked emission line profile variability (e.g. Wiggs \& Gies 1993, Rauw et al. 1999, Gosset et al. 2001...). Some wide WC + O binaries also exhibit episodic (recurrent) variability of their IR emission attributed to dust formation in the wind collision zone (see Williams 2002 for a review). Another distinctive signature of wind interactions is the excess X-ray emission produced in the shock-heated plasma. On statistical grounds, earlytype binaries are indeed found to be stronger X-ray sources than their single counterparts (Pollock 1987a, Chlebowski \& Garmany 1991). The $\mathrm{X}$-ray emission from colliding wind binaries is expected to display a significant phase-locked modulation (Pittard \& Stevens 1997) either as a consequence of a changing optical depth along the line of sight or as a result of a changing separation between the stars in eccentric binaries. Such variations have indeed been reported for a number of $\mathrm{O}+\mathrm{O}$ and WR + O binaries (e.g. Corcoran 1996, Willis et al. 1995, Rauw et al. 2002a).

In this chapter, I will focus on another aspect of the wind interaction in early-type binaries, i.e. the acceleration of relativistic particles near the hydrodynamical shock and the signatures of these particles throughout the spectra of these binaries. This chapter is organised as follows. Section 1 provides a brief introduction to the concept of wind - wind collisions and their potential role in the acceleration of relativistic particles. In Section 2, I consider the synchrotron radiation seen in the radio emission of some O and WR stars, whilst Sect. 3 is devoted to a discussion of the possible observational signature of relativistic particles at X-ray and $\gamma$-ray energies through inverse Compton scattering. Section 4 contains a brief overview of other mechanisms related to hot massive stars that may produce $\gamma$-ray radiation. Finally, the main conclusions as well as future perspectives are highlighted in Sect. 5 . 


\section{Some basic considerations}

\subsection{Wind interactions in early-type binaries}

Consider a system formed by two stars with spherically symmetric stellar winds (in the absence of the wind interaction). Let $\dot{\mathrm{M}}_{j}$ and $v_{\infty, j}$ be respectively the mass loss rate and the asymptotic wind velocity of star $j$. In a wide binary system, both winds reach their terminal velocity before they collide and the shape of the contact discontinuity is set by the wind momentum ratio defined as

$$
\mathcal{R}=\left(\frac{\dot{\mathrm{M}}_{1} v_{\infty, 1}}{\dot{\mathrm{M}}_{2} v_{\infty, 2}}\right)^{1 / 2}
$$

(see e.g. Eichler \& Usov 1993, Stevens et al. 1992). The intersection between the binary axis and the contact discontinuity lies at a distance $x^{\text {stag }}=\mathcal{R} d /(\mathcal{R}+1)$ from the center of mass of the star with the more energetic wind. Here $d$ stands for the orbital separation between the centers of the stars. In a binary system where both winds are of equal strength $(\mathcal{R}=1)$, the contact discontinuity can be approximated by a plane located mid-way between the two stars. In a more general situation with $\mathcal{R} \neq 1$, the contact discontinuity appears as a cone wrapped around the star with the less energetic wind (see Fig. 1).

Immediately behind the shock, the plasma is heated to a temperature of order

$$
T_{s, j}=\frac{3 \bar{m}_{j} v_{j}^{2}}{16 k}
$$

where $\bar{m}_{j}$ is the average mass per particle in the wind of star $j$ and $v_{j}$ stands for the pre-shock wind velocity (see e.g. Stevens et al. 1992). Due to the large pre-shock wind velocities, the post-shock plasma temperature is very high $\left(\geq 10^{7} \mathrm{~K}\right)$.

The properties of the post-shock gas depend to a large extent on the importance of radiative cooling that can be roughly estimated from the ratio between the characteristic cooling time and the typical escape time from the shock region:

$$
\frac{t_{\mathrm{cool}}}{t_{\mathrm{esc}}} \simeq \frac{v_{8}^{4} x_{12}^{\mathrm{stag}}}{\dot{M}_{-7}}
$$

where $v_{8}, x_{12}^{\text {stag }}$ and $\dot{M}_{-7}$ are respectively the pre-shock velocity in units $10^{3} \mathrm{~km} \mathrm{~s}^{-1}$, the distance to the contact surface in units $10^{7} \mathrm{~km}$ and the mass loss rate in units $10^{-7} \mathrm{M}_{\odot} \mathrm{yr}^{-1}$ (Stevens et al. 1992). If radiative cooling is important $\left(t_{\mathrm{cool}} / t_{\mathrm{esc}} \ll 1\right)$, the entire thermal energy produced in the shock is radiated away almost immediately. These highly radiative shocks collapse and the rather cool material in the interaction zone 
can contribute to the formation of optical emission lines. Otherwise, if $t_{\mathrm{cool}} / t_{\mathrm{esc}} \geq 1$, the shock region is essentially adiabatic.

Density

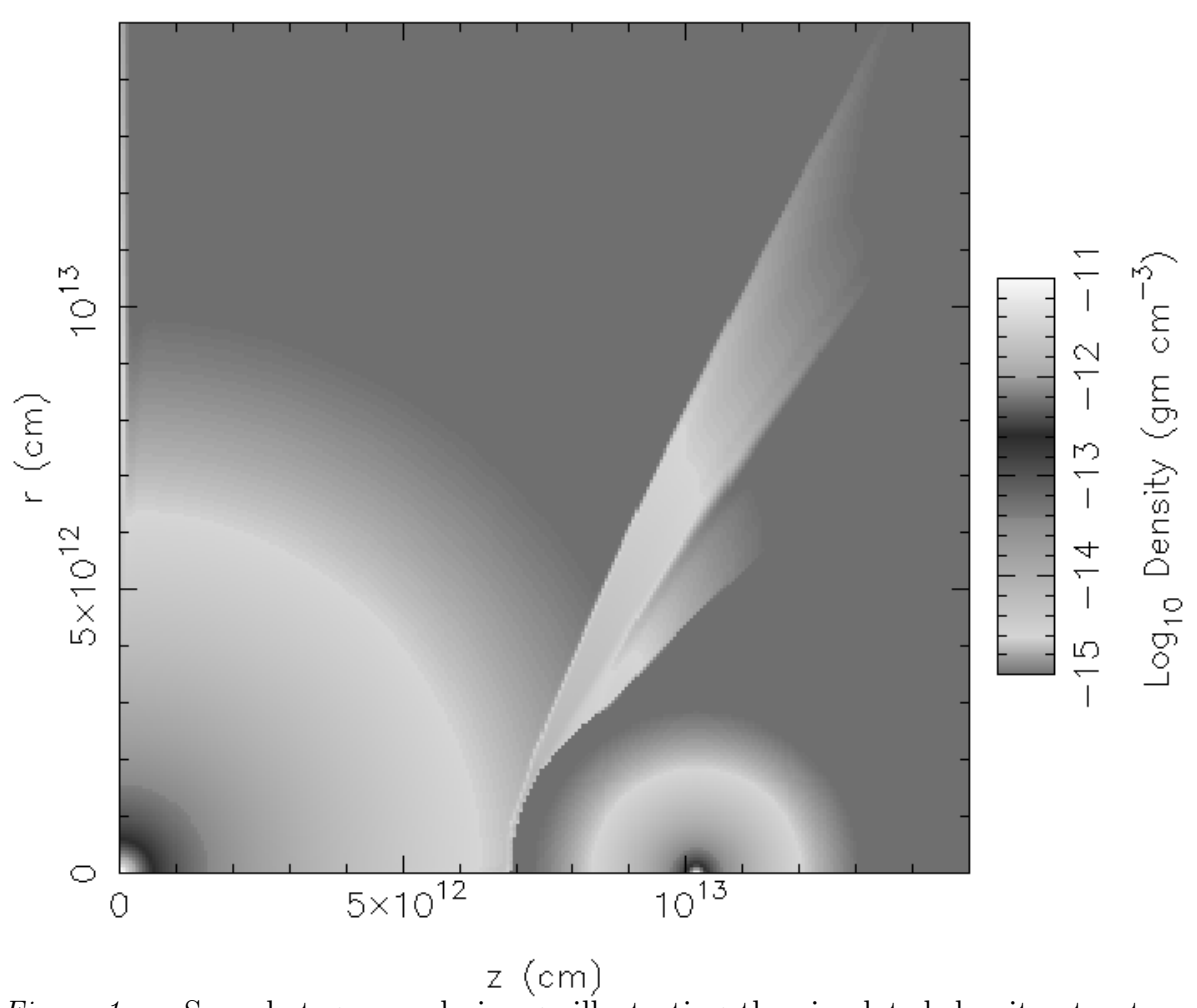

Figure 1. Snapshot gray-scale image illustrating the simulated density structure of an adiabatic wind collision. The center of mass of the primary lies at the origin of the axes, whereas the center of the secondary is located at $z \simeq 10^{13} \mathrm{~cm}$. The wind parameters are such that $\mathcal{R}=3.3$ and $\left(t_{\mathrm{cool}} / t_{\mathrm{esc}}\right)_{1}=7$ and $\left(t_{\mathrm{cool}} / t_{\mathrm{esc}}\right)_{2}=25$ for the primary and secondary post-shock winds respectively (Sana 2002, private communication).

An important feature associated with the wind collision in an earlytype binary is the fact that the shock can act as a site for particle acceleration (Eichler \& Usov 1993). In the following sections we will discuss the implications of the presence of relativistic particles in the winds of early-type stars and early-type binaries in particular.

\subsection{Relativistic particles in stellar winds}

Synchrotron radio emission has been observed for a number of earlytype stars (see Section 2 below). The detection of such a non-thermal 
emission from these stars implies that a population of relativistic particles is present in their winds. Let us consider what are the main ingredients required to account for this feature.

1.2.1 The acceleration of relativistic particles. Various scenarios have been suggested to explain the presence of relativistic electrons in the atmospheres of early-type stars. Most of them actually require the presence of hydrodynamical shocks. Indeed, free electrons and protons can be accelerated through the first order Fermi mechanism by crossing the shock front forth and back (see e.g. Bell 1978a, Longair 1992). As a result, relativistic electrons should have a power law differential energy distribution $N(\gamma) \propto \gamma^{-n}, \gamma$ being the Lorentz factor of the relativistic electrons. The spectral index $n$ is related to the shock compression ratio $\chi$ by

$$
n=\frac{\chi+2}{\chi-1}
$$

(Bell 1978a). If the shocked plasma radiates efficiently, the shock is quasi-isothermal and the compression ratio becomes very large. In this case one expects a value of $n \sim 1$. If instead the shock is adiabatic, then $\chi$ should not exceed 4 (for a strong shock) and $n \sim 2$.

Hydrodynamical shocks are found both in single and binary earlytype stars. For instance, in the case of single stars, the intrinsic instabilities of radiatively driven stellar winds (see e.g. Runacres \& Owocki 2002 and references therein) lead to the formation of shocks distributed throughout the stellar winds. Therefore, White (1985) and Chen \& White (1991a, 1994) suggested that relativistic electrons can be accelerated in the winds of single hot stars. In his model, White (1985) considered that relativistic particles accelerated in shocks in the inner wind region lose energy only through adiabatic expansion and could hence travel out to large distances. However, the interplay between relativistic electrons and the intense stellar radiation field implies strong energy losses through inverse Compton scattering and the electrons accelerated in the inner regions of the stellar wind cannot survive out to large radii. As a consequence, the relativistic electrons responsible for the radio synchrotron emission must be accelerated in situ, i.e. outside the radio photosphere (Chen \& White 1994, see also Sect. 2 below). Another consequence of the inverse Compton cooling might be a substantial non-thermal emission at X-ray and $\gamma$-ray energies (Pollock 1987b, Chen \& White 1991a, b).

In the case of massive binaries, particles can be accelerated through the Fermi mechanism in the shock region of the wind interaction zone (Eichler \& Usov 1993). An alternative scenario related to colliding wind 
binaries has been proposed by Jardine et al. (1996). These authors considered the current sheets that result from the collision of magnetized stellar winds. The compression of magnetic field lines in the current sheets leads to enhanced local field strengths and the resulting electric fields in the current sheet can accelerate particles also in the atmospheres of stars with rather modest surface magnetic fields.

Finally, one should note that the flow time associated with a shock inside a stellar wind is rather short (of the order of hours to a few days). Compared to the situation in a supernova remnant, where there is ample time to accelerate thermal particles to relativistic energies (Bell 1978b), the particles in a stellar wind spend less time in the vicinity of a shock. More theoretical work is therefore needed to check whether the mechanism proposed by Bell (1978b) for the initial acceleration of particles in supernova remnants works also in stellar winds. Also, while the relativistic electrons may well be injected with a power-law spectrum, various energy loss mechanisms (ionization losses, inverse Compton scattering, synchrotron losses...) will distort this distribution (see for instance the work of Melrose \& Crouch 1997 on the effect of synchrotron losses) and might even inhibit the acceleration of the electrons.

1.2.2 Magnetic fields. The question of the existence of magnetic fields in the stellar winds of early-type stars is still a controversial issue. Indeed, early-type stars do not have a convective zone beneath the photosphere that could generate a strong magnetic activity such as seen in the coronae of late-type stars. Non-thermal radio emission, if due to synchrotron radiation, is consistent with surface magnetic fields $\left(B_{*}\right)$ of the order of a hundred Gauss (Chen \& White 1994). However, the broad absorption lines in the spectra of OB stars render any direct determination of $B_{*}$ through Zeeman splitting extremely difficult. So far, magnetic fields have only been measured directly for a few early-type OB stars $\left(\sim 360 \mathrm{G}\right.$ in $\beta \mathrm{Cep}(\mathrm{B} 1 \mathrm{IV})$ and $\sim 1100 \mathrm{G}$ in $\theta^{1}$ Ori C $(\mathrm{O} 4-6 \mathrm{~V})$ see Donati et al. 2001, 2002). For most OB stars, the non-detection of the Zeeman effect puts an upper limit of order $\sim 100 \mathrm{G}$ on $B_{*}$ (Mathys 1999). In the case of WR stars, the optical spectra display broad emission lines that are formed throughout the stellar wind and no direct measurement of the magnetic fields of a WR star has been possible to date.

Indirect evidence for magnetic fields in early-type stars has been obtained from the rotational modulation of the stellar winds of some objects. In fact, the periodic variability seen in the spectra of some of these stars has been attributed to corotating structures in the stellar wind. One possibility to generate such corotating structures would be a 
stellar magnetic field. The field strength can be estimated by assuming that the outer radius of corotation of the stellar wind is equal to the Alfvén radius (see e.g. Rauw et al. 2001).

The existence of the stellar wind alters the geometry of the magnetic field lines of an otherwise dipolar field. Following the description of Eichler \& Usov (1993), the radial dependence of the magnetic field strength at large distance $r$ from the star should be described by a toroidal field:

$$
B(r)=B_{*} \frac{v_{\mathrm{rot}}}{v_{\infty}} \frac{R_{*}^{2}}{R_{\mathrm{A}} r}
$$

(see Fig. 2) where $R_{\mathrm{A}}$ is the Alfvén radius, $R_{*}$ the stellar radius and $v_{\text {rot }}$ is the rotation velocity at the stellar equator.

Magnetic fields are important both for the acceleration and emission mechanisms. For instance, the mean free path of a relativistic electron of energy $\gamma m_{e} c^{2}$ is given by

$$
\lambda(r) \propto \frac{\gamma m_{e} c^{2}}{e B(r)}
$$

(where $e$ is the charge of the electron and all parameters are expressed in Gaussian units) and this parameter has implications for the value of the cut-off energy of the relativistic electron distribution. In addition, the local magnetic field is of course an essential ingredient in the synchrotron emission process.

\section{Non-thermal radio emission from early-type stars}

The bulk of the radio emission from early-type stars is attributed to thermal free-free radiation produced in their stellar winds. Theoretical considerations indicate that these objects should have a radio spectral index ${ }^{1}$ of $\alpha \simeq 0.6$ (e.g. Wright \& Barlow 1975). Note that actual millimeter and radio observations suggest slightly larger spectral indices of $0.7-0.8$ for the free-free emission of WR and O stars (Williams 1996).

At radio wavelengths, the winds of early-type stars are optically thick and the optical depth at a radius $r$ is given through the following relation:

$$
\tau_{\nu}(r)=\int_{r}^{\infty} K(\nu, T) q\left(\frac{\dot{\mathrm{M}}}{4 \pi \mu m_{H} v_{\infty}}\right)^{2} \frac{d x}{x^{4}}
$$

where $K(\nu, T)$ is the linear free-free absorption coefficient (see Wright \& Barlow 1975, Leitherer et al. 1995), $q$ is the ratio between the electron and ion number densities, $\mu$ and $m_{H}$ are the mean atomic weight and the 


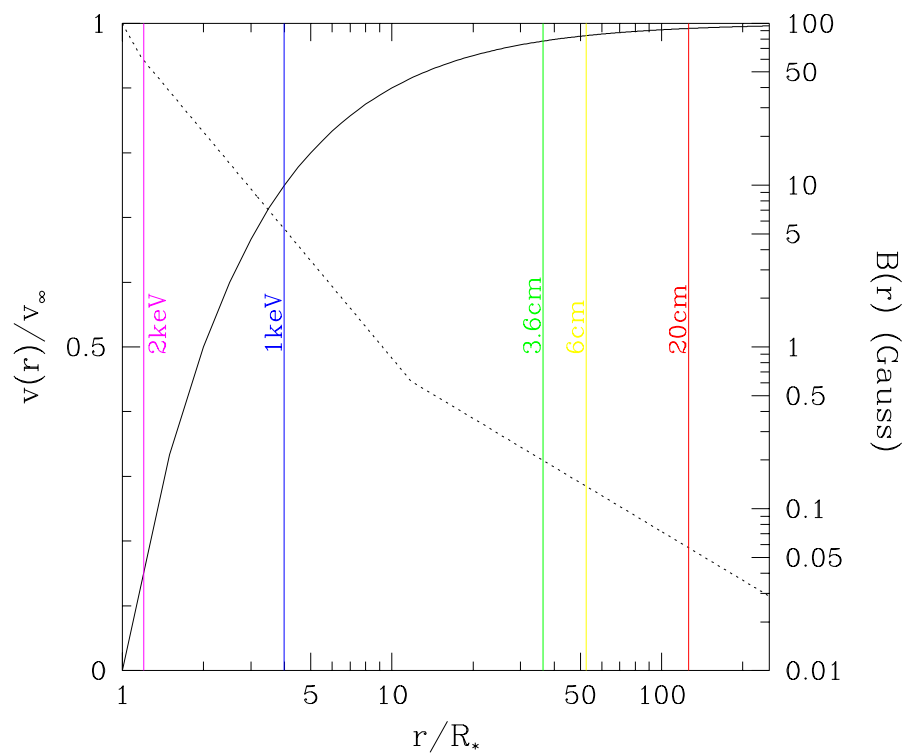

Figure 2. Illustration of the wind structure of a typical $\mathrm{O}$ star. The parameters are for $9 \operatorname{Sgr}\left(\dot{\mathrm{M}}=2.410^{-6} \mathrm{M}_{\odot} \mathrm{yr}^{-1}, v_{\infty}=2950 \mathrm{~km} \mathrm{~s}^{-1}, R_{*}=16 \mathrm{R}_{\odot}, v_{\mathrm{rot}}=250 \mathrm{~km} \mathrm{~s}^{-1}\right.$, see the references in Rauw et al. 2002b). The solid curve yields the wind velocity as a function of radius, assuming a so-called standard $\beta$ velocity law with $\beta=1$. The dotted line shows the radial dependence of the magnetic field assuming $B_{*}=100 \mathrm{G}$ and adopting the formalism of Eichler \& Usov (1993). The vertical lines indicate the location of surfaces of optical depth unity for various energies or wavelengths (given by the labels).

mass of the hydrogen atom respectively. For an isothermal $\mathrm{O}$ star wind of temperature $20000 \mathrm{~K}$ consisting mainly of ionized hydrogen with $\dot{\mathrm{M}}=$ $3 \times 10^{-6} \mathrm{M}_{\odot} \mathrm{yr}^{-1}$ and $v_{\infty}=2000 \mathrm{~km} \mathrm{~s}^{-1}$, we find that $\tau_{\nu}=1$ corresponds to radii of 850,1250 and $3000 \mathrm{R}_{\odot}$ (corresponding to about 50,75 and $\left.175 \mathrm{R}_{*}\right)$ at radio wavelengths of $3.6,6$ and $20 \mathrm{~cm}$ respectively. Note that these radii are even larger for Wolf-Rayet type stars. Therefore, any observable radio flux of an early-type star must arise in the outer regions of its stellar wind. In the case of massive binaries with spherically symmetric winds (outside of the colliding winds region), this condition translates into a critical value of the orbital period: below periods of the order of a month, the heavy wind opacity would prevent us from seeing any non-thermal radio radiation that might be produced in the shock front of a colliding wind WR + O binary (Eichler \& Usov 1993).

A subset of OB and WR stars display unusually high radio flux levels with spectral indices that deviate significantly $(\alpha \leq 0.0)$ from the canonical value expected for purely thermal free-free emission (e.g. Abbott et al. 1984, Bieging et al. 1989). This feature is attributed to a 


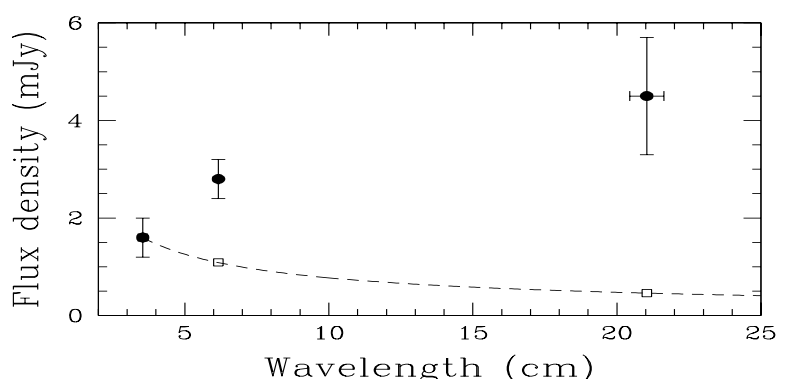

Figure 3. $\quad V L A$ radio fluxes of $9 \mathrm{Sgr}$ as reported by Rauw et al. (2002b). The observed fluxes are shown as filled circles. The dotted line and the open squares show the energy distribution that would be observed if the radio emission were thermal with a spectral index $\alpha=0.7$ (normalized to the observed flux at $3.6 \mathrm{~cm}$ ).

non-thermal, most probably synchrotron, emission component on top of the thermal wind emission. In fact, synchrotron radiation produced by relativistic particles should produce a power law spectrum with a spectral index $\alpha=-(n-1) / 2 \sim-0.5$. The non-thermal radio emission is often variable both in intensity and in spectral index (see e.g. Williams 1996). The observed synchrotron radiation is a definite sign of two things: (i) a magnetic field and (ii) a population of relativistic electrons must exist in the radio emitting region (i.e. outside the radio photosphere).

From an observational point of view, it is worth pointing out that most, if not all, of the WR stars that display a non-thermal radio emission are in fact binaries (see Dougherty \& Williams 2000). The situation is however less clear for non-thermal OB stars (see Table 1). While three stars out of eight are binary or triple systems, the multiplicity of the remaining five objects has not yet been fully investigated. However, at least in the case of $9 \mathrm{Sgr}$ (Rauw et al. 2002b) and HD 93250 (Rauw et al. 2003), observational hints towards multiplicity were found in the optical or X-ray domain.

HD $167971(\mathrm{O} 7.5 \mathrm{f}+\mathrm{O})$ has the strongest non-thermal radio emission among the OB stars detected in the survey of Bieging et al. (1989). Leitherer et al. (1987) describe HD 167971 as a close eclipsing O star binary $\left(\mathrm{P}_{\text {orb }}=3.32\right.$ days $)$ with a third more distant and much brighter Of companion (which could be a line of sight object).

Cyg OB2 \#5 is another multiple system. This system consists of a 6.6-day period close binary harboring an Of supergiant and a more evolved Ofpe/WN9 transition object (Rauw et al. 1999). A third star (probably early B-type) was found to lie 0.95" away from the close binary (see Contreras et al. 1997). The system displays a variable, partly non-thermal radio emission. The radio emission consists of two compo- 
nents: a primary associated with the close binary system and a secondary slightly elongated (non-thermal) component with a location consistent with emission from the bow shock between the winds of the close binary and the third component (Contreras et al. 1997). It is worth mentioning that the radio variability of the system is mainly due to the primary radio component (Dougherty 2000, private communication) that shifts between a purely thermal low state and a partly non-thermal high state on an apparent time scale of about 7 years (Miralles et al. 1994).

Table 1. Multiplicity of OB stars known to display a non-thermal radio emission.

\begin{tabular}{lclc}
\hline Star & Spect. type & Binarity & Reference \\
\hline HD 15558 & O5 III (f) & SB1 & {$[1]$} \\
HD 93250 & O3 V & X-ray variable & {$[2]$} \\
HD 164794 (=9 Sgr $)$ & O4 V $\left(\left(\mathrm{f}^{+}\right)\right)$ & RV variable & {$[3]$} \\
HD 168112 & O5 III & single? & - \\
HD 167971 & O7.5 f + O & triple & {$[4]$} \\
Cyg OB2 \#5 & O7 Ianf + Ofpe/WN9 & triple & {$[5]$} \\
Cyg OB2 \#8A & O6 Ib(f) & single? & - \\
Cyg OB2 \#9 & O5 f & single? & - \\
\hline
\end{tabular}

References: [1] Garmany \& Massey (1981), [2] Rauw et al. (2003), [3] Rauw et al. (2002b), [4] Leitherer et al. (1987), [5] Contreras et al. (1997).

The observed radio emission of an early-type star can be written

$$
S_{\nu}=S_{f f, \nu_{0}}\left(\frac{\nu}{\nu_{0}}\right)^{0.7}+A_{n t, \nu_{0}}(t)\left(\frac{\nu}{\nu_{0}}\right)^{\alpha(t)} \exp \left[-\tau_{\nu}(t)\right]
$$

Here $S_{f f, \nu_{0}}$ stands for the thermal emission due to the free-free process at frequency $\nu_{0}$, whereas $A_{n t, \nu_{0}}(t)$ and $\alpha(t)=-(n-1) / 2$ are respectively the non-thermal flux level at frequency $\nu_{0}$ and the spectral index. The latter two properties may display significant time variability. Likewise, if the position of the non-thermal source inside the wind changes, the optical depth $\tau_{\nu}$ due to free-free absorption towards the region of the non-thermal emission may also vary with time. Note that the spectral indices inferred from radio observations are rather steep, consistent with the electrons being accelerated in rather weak shocks. Finally, below a frequency $\nu_{R} \sim 20 n_{e} / B$ the synchrotron emission is cut off by the Razin effect that suppresses the beaming of the synchrotron radiation. Here $n_{e}$ stands for the electron density (Ginzburg \& Syrovatskii 1965).

Non-thermal radio emission is observed essentially in wide binaries where the shock region is located outside the radio photosphere. In 
close binaries, synchrotron emission could still be produced, but it might remain undetectable because of the optically thick wind. At least in the case of eccentric binaries, these considerations account for the observed phase-locked variability of the radio flux.

The most famous examples of non-thermal radio emitters associated with colliding wind WR + O binaries are probably WR 140 (WC7 + O5, e.g. Williams et al. 1990, White \& Becker 1995) and WR 147 (e.g. Williams et al. 1997).

WR 140 has a highly eccentric $(e=0.84)$ orbit with a period of $7.9 \mathrm{yrs}$ and the non-thermal radio flux displays a prominent phase-locked variation (e.g. White \& Becker 1995). Between phases 0.4 and 0.95 (phase 0.0 corresponding to periastron passage), the emission at $6 \mathrm{~cm}$ increases dramatically displaying a broad maximum around phase 0.8 . The higher frequency emissions rise, peak and decline somewhat earlier than emission at longer wavelengths (White \& Becker 1995). The shape of the radio light curve at different frequencies results from a combination of two effects (Williams et al. 1990, 1994). First, the region of the wind interaction is burried deep inside the huge radio photosphere of the WR star, except at phases away from periastron when the shock zone is farther away from the WR core and eventually moves out of the WR's radio photosphere. In addition, as a result of the cavity produced by the shock cone in the WR wind, the non-thermal radio emission becomes observable when the $\mathrm{O}$ star with its lower density (and thus less opaque) wind passes in front of its WR companion.

WR 147 is a visual binary consisting of a WN8 star and a late-O, earlyB type companion. The radio emission from this system was resolved by MERLIN into a thermal component associated with the wind of the WN8 star and a non-thermal component located in between the WR star and its visual companion (see Williams et al. 1997). The location of the nonthermal component (closer to the OB star) as well as its morphology are consistent with the expectations of a colliding wind scenario (Dougherty et al. 2003). Interestingly, WR 147 is also the first early-type system where high resolution Chandra ACIS-I observations provided evidence for a spatially extended X-ray emission. The X-rays are found to peak close to the position of the radio bow shock (Pittard et al. 2002).

\section{Inverse Compton scattering}

Synchrotron radio-emission is expected to account only for a fraction of the total non-thermal energy budget. In fact, given the enormous 
supply of photospheric UV photons in the winds of OB stars, inverse Compton (IC) scattering becomes a major energy loss mechanism for relativistic electrons and non-thermal X-ray and low-energy $\gamma$-rays should be generated, resulting approximately in a power law spectrum from $\mathrm{keV}$ to MeV energies (Pollock 1987b, Chen \& White 1991a,b). In the Thomson regime, the average energy of a photon after inverse Compton scattering with respect to its initial energy is of order $h \nu_{I C} \sim \frac{4}{3} \gamma^{2} h \nu_{*}$. Given the average photon energy $h \nu_{*}$ of the radiation field of a typical early-type star, Lorentz factors of order $10^{2}-10^{4}$ are sufficient to produce IC X-ray and $\gamma$-ray radiation. The spectrum resulting from IC scattering by a power law electron distribution of index $n$ is a power law of photon index $\Gamma=(n+1) / 2$. The critical question is whether the IC $\mathrm{X}$-ray and $\gamma$-ray flux levels are high enough to be detected.

In their model, Chen \& White (1991a) considered electrons accelerated in strong wind instability shocks with $\chi=4$ giving $n=2$ and $\Gamma=1.5$. These shocks are distributed throughout the wind of single $\mathrm{O}$ stars and these authors therefore treated the IC process in the isotropic approximation. Note that in the case of single stars, the IC X-ray, $\gamma$-ray and synchrotron radio emission are not necessarily correlated because they arise in physically distinct regions. Indeed, whilst photoelectric absorption by the circumstellar material can affect the X-ray spectra of early-type stars up to energies of $\sim 1 \mathrm{keV}$, it has little impact on the higher energy X-rays. Therefore, the optical depth to hard X-rays and soft $\gamma$-rays throughout the wind is small ${ }^{2}$. Moreover, inverse Compton scattering is expected to be most efficient within a few stellar radii where the UV radiation density is highest. On the other hand, as a consequence of the large free-free optical depth at radio frequencies, radio synchrotron emission can only be observed from the outer regions of the wind (beyond about fifty stellar radii, see Fig. 2). The properties of the expected non-thermal emission of single stars at different energies thus reflect the radial distribution of the relativistic electron spectrum throughout the wind.

Chen \& White (1991b) investigated the $\gamma$-ray luminosities due to inverse Compton scattering in the atmospheres of O stars. These authors estimated total IC luminosities of $\sim 810^{33}$ and $6310^{33} \mathrm{erg} \mathrm{s}^{-1}$ for the O4 stars $\zeta$ Pup and 9 Sgr respectively. However, their predictions depend strongly on the interpretation of the hard X-ray emission (at energies above $3 \mathrm{keV}$ ) seen in $\zeta$ Ori with the Solid State Spectrometer onboard the EINSTEIN observatory (see however below).

In a colliding wind situation, the shock region is located closer to the surface of the star with the less energetic wind (see Fig. 1). Therefore, 
the seed photons for inverse Compton scattering will mainly come from the UV radiation of this star. In this case, it is the same population of relativistic electrons (accelerated in the wind interaction zone) that produces the synchrotron and IC emission. The inverse Compton scattering emission must be computed in the non-isotropic case (using for instance the head-on approximation, see Reynolds 1982, Mücke \& Pohl 2002).

\subsection{Non thermal X-ray emission from early-type stars?}

Rauw et al. (2002b) performed a multi-wavelength investigation of 9 Sgr. Their VLA data obtained simultaneously with an XMM-Newton observation indicate a definite non-thermal radio emission (see Fig. 3). The X-ray spectra of $9 \mathrm{Sgr}$ reveal a hard X-ray component that can be fitted equally well by a multi-temperature thermal model including some plasma at $\mathrm{kT} \geq 1.46 \mathrm{keV}$ or by a rather steep power law $(\Gamma \geq 2.9)$ component (see Fig. 4). When interpreted in the framework of the theory of Bell (1978a), such a steep power law corresponds to acceleration by shocks with a low compression ratio $(\chi \leq 1.8)$.

The 9 Sgr data discussed by Rauw et al. (2002b) provide the tightest constraints so far on the properties of a hard X-ray power law tail in an $\mathrm{O}$ star. In fact, the EINSTEIN-SSS observations of the Orion Belt stars that motivated the work of Chen \& White (1991a) may have been affected by calibration issues involving the formation of ice on the instrument entrance window and subsequent $A S C A$ observations of $\delta$ Ori failed to confirm the existence of a hard power law tail in its X-ray spectrum (Corcoran et al. 1994).

It is worth pointing out that the $V L A$ observations of 9 Sgr also suggest a low compression ratio of $\chi \simeq 1.7$. In this context, it must be stressed that the compression ratios inferred from the X-ray and radio data should be seen as typical or average values. Rather than having a unique compression ratio, the shocks in the wind are likely to have a distribution of compression ratios, as is also suggested by hydrodynamical models.

Interestingly, Skinner et al. (2002) reported the existence of a hard Xray component in the $X M M$-EPIC spectra of the presumably single WolfRayet star WR 110. This hard component could be either thermal $(\mathrm{kT} \geq$ $3 \mathrm{keV}$ ) or non-thermal emission produced by inverse Compton scattering. In the former case, the hard X-rays could arise in a wind collision zone of a $\mathrm{WR}+\mathrm{O}$ binary with an as yet undetected $\mathrm{O}$ star companion. In the latter hypothesis, the hard component can be fitted with a photon index 
$\Gamma=2.2$, not too different from the theoretical value for strong adiabatic shocks $(\Gamma \sim 1.5$, see above). The radio emission from WR 110 yields no indication of a synchrotron component. However, the lack of nonthermal radio emission does not rule out the existence of a non-thermal $\mathrm{X}$-ray emission since, as pointed out above, the two phenomena may not be correlated if the synchrotron radio emission forms deep within the wind where it would be strongly absorbed by the huge free-free opacity.
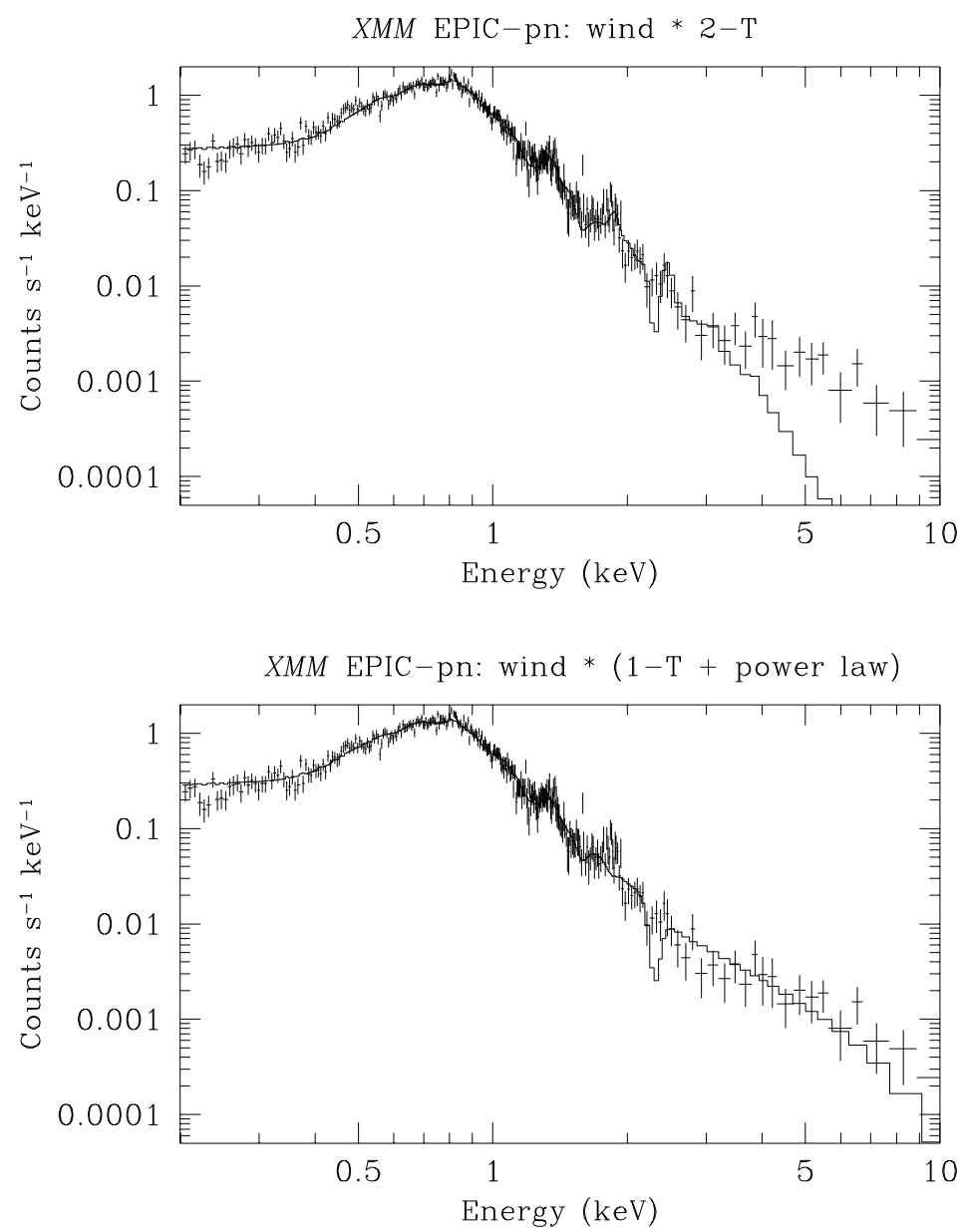

Figure 4. X-ray spectrum of 9 Sgr as observed with the EPIC-pn instrument onboard XMM - Newton. The upper panel illustrates the fit of the spectrum with a 2-T thermal plasma model with proper wind absorption cross sections. The model fails to reproduce the hard tail of the spectrum (beyond $\sim 3 \mathrm{keV}$ ). A better fit is obtained with a 1-T thermal model + non-thermal power law component as shown in the lower panel. For details see Rauw et al. (2002b). 


\subsection{A possible connection with unidentified $\gamma$-ray sources?}

At this stage, it is interesting to briefly consider the overall distribution of early-type stars in our Galaxy. The latest version of the Catalog of Galactic O Stars (Cruz-González et al. 1995) lists a total of 644 known O stars of luminosity class V. As far as WR stars are concerned, the VIIth Catalogue of Galactic Wolf-Rayet Stars (van der Hucht 2001) includes 227 entries. The actual total number of early-type stars in the Milky Way is certainly much larger: many hot massive stars are expected to populate the highly obscured inner regions of the Galaxy. Reed (2000) and van der Hucht (2001) estimated the total Galactic population of O-B2 stars and WR stars at respectively $\sim 60000$ and $\sim 6500$.

Gies (1987) investigated the kinematical and binary properties of an essentially complete sample of 195 Galactic O-type stars brighter than $V=8.0 .80 \%$ of the stars in this sample are found at a Galactic latitude $\left|b_{I I}\right| \leq 5^{\circ}$ and their distribution traces the spiral arms of the Galaxy. Gies also showed that $70 \%$ of all stars in his sample are members of open clusters or $\mathrm{OB}$ associations. In a subsequent study, Gies et al. (1998) found that among the O stars in clusters and associations, 75\% are either visual or spectroscopic binaries. The fraction of binaries is significantly lower among field stars and only a small fraction of binaries was found among runaway $\mathrm{O}$ stars. The situation is less clear among WR stars where considerable observational biases affect the binary census. Nevertheless, van der Hucht (2001) estimated the binary fraction among WR stars at $\sim 39 \%$ (including probable binaries).

In summary, there exists a large number of single and binary earlytype stars distributed over the Galactic Plane. Whether or not they produce a detectable emission at $\gamma$-ray energies strongly depends on the parameters of the individual systems.

From an observational point of view, many of the yet unidentified EGRET sources must be of Galactic origin and appear correlated with the massive stars population (e.g. Montmerle 1979, Manchanda et al. 1996, Romero et al. 1999).

Romero et al. (1999) studied the possible association between sources in the third EGRET catalogue (Hartman et al. 1999) and catalogues of WR and Of stars, supernova remnants (SNRs) and OB association (believed to be essentially pulsar tracers). Marginally significant correlations were found for the WR and Of stars, whereas highly significant correlations were found for SNRs and OB associations. An a posteriori analysis led Romero et al. to conclude further that among the positive 
correlations with massive stars, WR 142, WR 140 and Cyg OB2\#5 were most likely actual $\gamma$-ray sources associated respectively with the sources 3EG J2021+3716, 3EG J2022+4317 and 3EG J2033+4118.

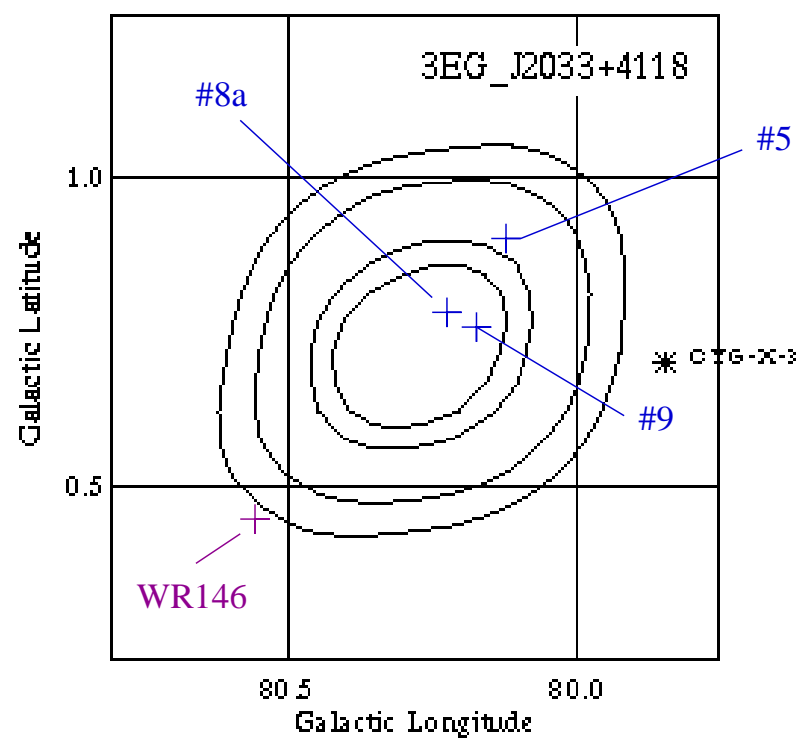

Figure 5. Location of the EGRET source 3EG J2033+4118 in Galactic coordinates (from Hartman et al. 1999). The contours illustrate 50\%, 68\%, 95\% and 99\% statistical probability that a single source lies within the given contour. The plus signs indicate the positions of the three Cyg OB2 stars that display a non-thermal radio emission.

Let us consider the case of 3EG J2033+4118 a bit more in detail. This source lies at $l_{I I}=80.27^{\circ}$ and $b_{I I}=0.73^{\circ}$ and has a flux of $(7.3 \pm 0.7) 10^{-7}$ photons $\mathrm{cm}^{-2} \mathrm{~s}^{-1}$ at energies above $100 \mathrm{MeV}$ with a photon index of $\Gamma=1.96 \pm 0.10$ (Hartman et al. 1999). Chen et al. (1996) studied the possible connection between this source and the Cyg OB2 association. Cyg X-3 lies just outside the error box of the source (see Fig. 5), but the $\gamma$-ray flux does not display the $4.8 \mathrm{hrs}$ period of $\mathrm{Cyg} \mathrm{X}-3$ and it seems therefore more likely that it is associated with the Cyg OB2 stars. Assuming the source lies at the distance of Cyg OB2, it should have a luminosity of $2.410^{35} \mathrm{erg} \mathrm{s}^{-1}$ in the EGRET energy range. It is extremely interesting that three Cyg OB2 stars displaying non-thermal radio emission fall into the $95 \%$ confidence contour (Fig. 5). These are Cyg OB2 \#5 (see below), \#8a (Waldron et al. 1998) and \#9 (e.g. Phillips \& Titus 1990).

Benaglia et al. (2001) studied the possible contribution of the triple system Cyg OB2 \#5 to 3EG J2033+4118. The authors found that in- 
verse Compton scattering of UV photons by relativistic electrons $(\gamma \sim$ $310^{3}-410^{4}$ ) accelerated in the wind interaction zone between the close binary and the nearby optical companion is likely to be the main contributor to the $\gamma$-ray emission of the system, accounting for a luminosity of $\sim 810^{34} \mathrm{erg} \mathrm{s}^{-1} \cdot \pi^{0}$ decay of relativistic protons accelerated in the inner regions of the winds of the close binary (see Sect. 4) could contribute another $\sim 510^{34} \mathrm{erg} \mathrm{s}^{-1}$. Due to the lower cut-off energy $\left(\gamma \leq 10^{3}\right)$, inverse Compton scattering in the close binary system probably does not play an important role in the $\gamma$-ray emission at EGRET energies, it might however contribute at lower energies (i.e. in the INTEGRAL energy range).

As another example, Benaglia \& Romero (2003) investigate the relation between the 3EG J2022+4317 EGRET source and the colliding wind binary WR 140. The EGRET source has a flux of $(24.7 \pm 5.2) 10^{-8}$ photons $\mathrm{cm}^{-2} \mathrm{~s}^{-1}$ and a photon index of $\Gamma=2.31 \pm 0.19$. Assuming it lies at the distance of WR 140, this flux corresponds to a luminosity of $3.210^{34} \mathrm{erg} \mathrm{s}^{-1}$. WR 140 is the only high energy source in the vicinity of this EGRET source. Benaglia \& Romero estimate total IC luminosities between $100 \mathrm{MeV}$ and $20 \mathrm{GeV}$ of about $2.110^{34} \mathrm{erg} \mathrm{s}^{-1} \cdot \pi^{0}$ decay from relativistic protons interacting with the HI bubble found near WR 140 could provide an additional extended emission contributing to the EGRET detection.

\subsection{Variability}

Since the IC process in a colliding wind binary is anisotropic, variability of the $\gamma$-ray emission could arise from the fact that the emitted power per volume element is dependent on the scattering angle. As the two stars of the binary revolve around each other, the angle under which the interaction zone is seen changes, therefore leading to a modulation of the observable IC $\gamma$-ray emission. Mücke \& Pohl (2002) first modelled this effect for a wide WR + O colliding wind system. Adopting the geometrical model of Eichler \& Usov (1993) and assuming an injection particle spectrum $\propto \gamma^{-n}$, these authors demonstrate that the observed $\gamma$-ray flux could vary by an order of magnitude.

An additional variability of the IC radiation at $\gamma$-ray energies could result from the orbital modulation of the UV flux in the case of an eccentric colliding wind binary. 


\section{Other $\gamma$-ray emission mechanisms}

Several other mechanisms related to early-type stars have been proposed to produce observable amounts of $\gamma$-ray emission.

White \& Chen (1992) proposed that a small fraction of thermal ions could be accelerated through the first order Fermi mechanism to relativistic energies by the shocks embedded in the highly unstable radiatively driven winds. These relativistic ions interact with the thermal ions to produce $\gamma$-rays via $\pi^{0}$ decay with an energy peak around $67 \mathrm{MeV}$. These authors estimated that individual Cyg OB2 stars could be marginally detectable with EGRET, whilst the combined $\gamma$-rays from the Cyg OB2 cluster could be substantial. However, the number density of non-thermal ions (and therefore the production rate of $\gamma$-rays at its peak energy) depend strongly on the poorly constrained $B_{*}$. In fact, in this model, the high energy cut-off of the particle distribution is quite sensitive to the maximum particle mean free path $\left(\propto B^{-1}\right)$.

Montmerle (1979) showed that about half of the unidentified COS B sources lie in regions harboring young objects such as SNRs and OB associations. In his scenario, high energy protons are injected by young stars into SNRs where they undergo Fermi acceleration up to relativistic energies. These relativistic protons subsequently interact with the protons in the parent molecular cloud of the OB association to produce a diffuse $\gamma$-ray emission through $\pi^{0}$ decay. Alternatively, Cassé \& Paul (1980) argued that particles could be accelerated at the terminal shock of the stellar winds without the need of a SNR.

Manchanda et al. (1996) suggested that the combined winds of the stars in young open clusters interact with the gas in the cluster therefore creating a system of bow shocks at the interface between cluster members. At these shocks, protons could be accelerated to relativistic velocities and produce $\gamma$-ray emission through $\pi^{0}$ decay.

Finally, Pollock (1987b) proposed that $\gamma$-rays may also be produced through bremsstrahlung in the dense winds of WR stars.

\section{Conclusions and future perspectives}

Synchrotron radio emission observed from a subset of the early-type stars forms only the tip of the non-thermal iceberg. As outlined in this chapter, relativistic electrons most probably interact with the intense UV radiation field of these stars to generate a substantial inverse Compton radiation at $\mathrm{X}$-ray and $\gamma$-ray energies. The latter radiation may be detectable with the INTEGRAL and GLAST observatories. In fact, Benaglia \& Romero (2003) evaluated the expected $\gamma$-ray fluxes in the energy bands of these satellites for three colliding wind binaries (WR 140, 
WR 146, WR 147). WR 140 and WR 147 were estimated to have fluxes of $8.310^{-4}$ and $1.210^{-3}$ photons $\mathrm{cm}^{-2} \mathrm{~s}^{-1}$ in the $15 \mathrm{keV}-10 \mathrm{MeV}$ IBIS sensitivity range and $1.110^{-6}$ and $9.410^{-7}$ photons $\mathrm{cm}^{-2} \mathrm{~s}^{-1}$ in the $20 \mathrm{MeV}$ - $300 \mathrm{GeV} G L A S T$ sensitivity range. For instance, according to the $I N$ TEGRAL Observing Time Estimator, WR 140 and WR 147 could be detected at the $3 \sigma$ level in the $30-50 \mathrm{keV}$ range within an exposure time of $15 \mathrm{ksec}$ with the IBIS imager. With its unprecedented angular resolution, INTEGRAL is especially well suited to disentangle the IC emission from individual early-type stars in open clusters. Many new exciting results are therefore expected from the ongoing Galactic Plane Survey observing programme of the INTEGRAL satellite.

Finally, let us stress that the simultaneous observation of synchrotron radio emission and IC $\gamma$-ray emission of colliding wind binaries offers the exciting perspective of determining the magnetic field strength at the location of the wind interaction zone which can be related back to the surface field.

\section{Notes}

1. The radio spectral index is defined by $F_{\nu} \propto \nu^{\alpha}$.

2. Note however that high-energy $\gamma$-ray photons in the $\mathrm{TeV}$ energy range could most probably not escape the base of the stellar winds because they would interact with the stellar photons causing electron/positron pair production: $\gamma+\gamma \rightarrow e^{+}+e^{-}$(see Reimer 2003).

\section{Acknowledgments}

It is a pleasure to thank Professors G.E. Romero and K.S. Cheng for their invitation to contribute this chapter. I wish to express my gratitude to my colleagues from the Groupe d'Astrophysique des Hautes Energies in Liège and to Drs. R. Blomme (Brussels), A. Reimer (Bochum) and A. Pollock (ESA) for discussion. My thanks go also to the Fonds National de la Recherche Scientifique (Belgium) for multiple assistance. This research is also supported in part by contracts $\mathrm{P} 4 / 05$ and $\mathrm{P} 5 / 36$ "Pôle d'Attraction Interuniversitaire" (SSTC-Belgium) and through PRODEX grants linked to the ESA XMM-Newton and INTEGRAL missions.

\section{References}

Abbott, D.C., Bieging, J.H., \& Churchwell, E.B. 1984, ApJ, 280, 671

Bell, A.R. 1978a, MNRAS, 182, 147

Bell, A.R. 1978b, MNRAS, 182, 443

Benaglia, P., Romero, G.E., Stevens, I.R., \& Torres, D.F. 2001, A\&A, 366, 605

Benaglia, P., \& Romero, G.E. 2003, A\&A, 399, 1121

Bieging, J.H., Abbott, D.C., \& Churchwell, E.B. 1989, ApJ, 340, 518

Cassé, M., \& Paul, J.A. 1980, ApJ, 237, 236 
Chen, W., \& White, R.L. 1991a, ApJ, 366, 512

Chen, W., \& White, R.L. 1991b, ApJ, 381, L63

Chen, W., \& White, R.L. 1994, Ap\&SS, 221, 259

Chen, W., White, R.L., \& Bertsch, D. 1996, A\&AS, 120, 423

Cherepashchuk, A.M. 1976, Sov.Ast. Lett., 2, 138

Chlebowski, T., \& Garmany, C.D. 1991, ApJ, 368, 241

Contreras, M.E., Rodríguez, L.F., Tapia, M., Cardini, D., Emanuele, A., Badiali, M., \& Persí, P. 1997, ApJ, 488, L153

Corcoran, M.F. 1996, Rev. Mex. Astron. Astrof. Conf. Series, 5, 54

Corcoran, M.F., Waldron, W.L., MacFarlane, J.J., et al. 1994, ApJ, 436, L95

Cruz-González, C., Recillas-Cruz, E., Costero, R., \& Peimbert, M. 1995, VizieR Online Data Catalog: III/84B, originally published in 1974, Rev. Mex. Astron. Astrof., 1,211

Donati, J.-F., Wade, G.A., Babel, J., Henrichs, H.F., de Jong, J.A., \& Harries, T.J. 2001, MNRAS, 326, 1265

Donati, J.-F., Babel, J., Harries, T.J., Howarth, I.D., Petit, P., \& Semel, M. 2002, MNRAS, 333, 55

Dougherty, S.M., \& Williams, P.M. 2000, MNRAS, 319, 1005

Dougherty, S.M., Pittard, J.M., Kasian, L., Coker, R.F., Williams, P.M., \& Lloyd, H.M. 2003, A\&A, 409, 217

Eichler, D., \& Usov, V.V. 1993, ApJ, 402, 271

Garmany, C.D., \& Massey, P. 1981, PASP, 93, 500

Gies, D.R., 1987, ApJS 64, 545

Gies, D.R., Hartkopf, W.I., Mason, B.D., Bagnuolo, W.G.Jr., ten Brummelaar, T., \& McAlister, H.A. 1998, in Boulder-Munich II: Properties of Hot Luminous Stars, ed. I.D. Howarth, ASP Conf. Series, 131, 382

Ginzburg, V.L., \& Syrovatskii, S.I. 1965, ARA\&A, 3, 297

Gosset, E., Royer, P., Rauw, G., Manfroid, J., \& Vreux, J.-M. 2001, MNRAS, 327, 435

Hartman, R.C., Bertsch, D.L., Bloom, S.D., et al. 1999, ApJS, 123, 79

Jardine, M., Allen, H.R., \& Pollock, A.M.T. 1996, A\&A, 314, 594

Lebedev, M.G., \& Myasnikov, A.V. 1988, in Numerical Methods in Aerodynamics, eds. V.M. Paskonov \& G.S. Roslyakov, Moscow State University Press, Moscow, 3

Leitherer, C., Forbes, D., Gilmore, A.C., et al. 1987, A\&A, 185, 121

Leitherer, C., Chapman, J.M., \& Koribalski, B. 1995, ApJ, 450, 289

Longair, M.S. 1992, High Energy Astrophysics, Vol. 1: Particles, Photons and their Detection, Cambridge University Press

Luo, D., McCray, R., \& MacLow, M.-M. 1990, ApJ, 362, 267

Manchanda, R.K., Polcaro, V.F., Norci, L., et al. 1996, A\&A, 305, 457

Mathys, G. 1999, in Variable and Non-spherical Stellar Winds in Luminous Hot Stars, eds. B. Wolf, O. Stahl, \& A.W. Fullerton, Lecture Notes in Physics, 523, 95

Melrose, D., \& Crouch, A. 1997, Publ. Astron. Soc. Aust., 14, 251

Miralles, M.P., Rodríguez, L.F., Tapia, M., Roth, M., Persí, P., Ferrari-Toniolo, M., \& Curiel, S. 1994, A\&A, 282, 547

Montmerle, T. 1979, ApJ, 231, 95

Mücke, A., \& Pohl, M. 2002, in Interacting Winds from Massive Stars, eds. A.F.J. Moffat \& N. St-Louis, ASP Conf. Series, 260, 355

Phillips, R.B., \& Titus, M.A. 1990, ApJ, 359, L15

Pittard, J.M., \& Stevens, I.R. 1997, MNRAS, 292, 298 
Pittard, J.M., Stevens, I.R., Williams, P.M., Pollock, A.M.T., Skinner, S.L., Corcoran, M.F., \& Moffat, A.F.J. 2002, A\&A, 388, 335

Pollock, A.M.T. 1987a, ApJ, 320, 283

Pollock, A.M.T. 1987b, A\&A, 171, 135

Prilutskii, O., \& Usov., V. 1976, Sov.Ast.-AJ, 20, 2

Rauw, G., Vreux, J.-M., \& Bohannan, B., 1999, ApJ, 517, 416

Rauw, G., Morrison, N.D., Vreux, J.-M., Gosset, E., \& Mulliss, C.L. 2001, A\&A, 366, 585

Rauw, G., Vreux, J.-M., Stevens, I.R., Gosset, E., Sana, H., Jamar, C., \& Mason, K.O. 2002a, A\&A, 388, 552

Rauw, G., Blomme, R., Waldron, W.L., et al. 2002b, A\&A, 394, 993

Rauw, G., Vreux, J.-M., Antokhin, I.I., et al. 2003, in New Visions of the X-ray Universe in the XMM-Newton and Chandra Era, ed. F. Jansen, ESA SP-488, in press

Reed, B.C. 2000, AJ, 120, 314

Reimer, A. 2003, in Proceedings of the 28th International Cosmic Ray Conference, in press

Reynolds, S.P. 1982, ApJ, 256, 38

Romero, G.E., Benaglia, P., \& Torres, D.F. 1999, A\&A, 348, 868

Runacres, M.C., \& Owocki, S.P. 2002, A\&A, 381, 1015

Skinner, S.L., Zhekov, S.A., Güdel, M., \& Schmutz, W. 2002, ApJ, 572, 477

Stevens, I.R., Blondin, J.M., \& Pollock, A.M.T. 1992, ApJ, 386, 265

van der Hucht, K.A. 2001, New Astron. Reviews, 45, 135

Waldron, W.L., Corcoran, M.F., Drake, S.A., \& Smale, A.P. 1998, ApJS, 118, 217

White, R.L. 1985, ApJ, 289, 698

White, R.L., \& Becker, R.H. 1995, ApJ, 451, 352

White, R.L., \& Chen, W. 1992, ApJ, 387, L81

Wiggs, M.S., \& Gies, D.R. 1993, ApJ, 407, 252

Williams, P.M. 1996, in Radio Emission from the Stars and the Sun, eds. A.R. Taylor \& J.M. Paredes, ASP Conf. Series, 93, 15

Williams, P.M. 2002 in Interacting Winds from Massive Stars, eds. A.F.J. Moffat \& N. St-Louis, ASP Conf. Series, 260, 311

Williams, P.M., van der Hucht, K.A., Pollock, A.M.T., Florkowski, D.R., van der Woerd, H., \& Wamsteker, W.M. 1990, MNRAS, 243, 662

Williams, P.M., van der Hucht, K.A., \& Spoelstra, T.A.T. 1994, A\&A, 291, 805

Williams, P.M., Dougherty, S.M., Davis, R.J., van der Hucht, K.A., Bode, M.F., \& Setia Gunawan, D.Y.A. 1997, MNRAS, 289, 10

Willis, A.J., Schild, H., \& Stevens, I.R. 1995, A\&A, 298, 549

Wright, A.E., \& Barlow, M.J. 1975, MNRAS, 170, 41 